\title{
A DECOMPOSITION METHOD FOR A SEMILINEAR BOUNDARY VALUE PROBLEM WITH \\ A QUADRATIC NONLINEARITY
}

\author{
MICHAEL S. GORDON
}

Received 17 May 2004 and in revised form 11 January 2005

The author adapts the decomposition method of Adomian to find a series solution of a one-dimensional boundary value problem for a semilinear heat equation with a quadratic nonlinearity. Local and global convergence results are obtained.

\section{Introduction}

In this work, we consider the following semilinear boundary value problem for $u(x, t)$ on the interval $0<x<\pi, t>0$ :

$$
\begin{gathered}
\partial_{t} u=\partial_{x x} u+\gamma u^{2}, \\
u(0, t)=u(\pi, t)=0, \\
u(x, 0)=f(x),
\end{gathered}
$$

where $f \in C([0, \pi])$.

The method of solution is based heavily on Adomian's method [1] of writing the solution as a series,

$$
u(x, t)=\sum_{n=1}^{\infty} u_{n}(x, t)
$$

and reducing the problem to one of iteratively solving a linear equation for $u_{n}$ once the previous iterates have been determined. However, Adomian partitions (1.4) into a sequence of linear ODEs in either $x$ or $t$ whose solutions cannot generally be made to satisfy the boundary and initial conditions. Even when applied to an initial value problem with boundary conditions, the convergence of the solution depends sensitively on powers of $f$ and its derivatives. In solving a similar problem in [1], Adomian must choose very specific initial data to guarantee local convergence in time. Our method arranges terms so that each linear problem is a PDE boundary value problem which is naturally solved with an expansion of eigenfunctions of $\partial_{x x}$ or a similar operator. We are able to show local convergence for any initial data $f$ and global convergence given a suitable bound 
on $f$. The method is similar to that of [2] which uses decomposition to derive special eigenfunction expansion solutions of (1.1) and (1.2).

In Section 2, we outline the method and construct a series solution of (1.1), (1.2), and (1.3), and in Section 3, we prove local and global existence results for the solution. In Section 4, we construct a series solution of (1.1) and (1.3) with Neumann boundary conditions in place of (1.2) and prove a local convergence result for the solution.

\section{The method}

To begin, (1.4) is substituted into (1.1) to obtain

$$
\sum_{n=1}^{\infty} \partial_{t} u_{n}=\sum_{n=1}^{\infty} \partial_{x x} u_{n}+\gamma \sum_{n=2}^{\infty} \sum_{k=1}^{n-1} u_{n-k} u_{k}
$$

Based on this, we seek solutions to the sequence of equations

$$
\begin{gathered}
\partial_{t} u_{1}=\partial_{x x} u_{1} \\
\partial_{t} u_{n}=\partial_{x x} u_{n}+\gamma \sum_{k=1}^{n-1} u_{n-k} u_{k}, \quad n \geq 2
\end{gathered}
$$

Notice that, once $u_{1}, u_{2}, \ldots, u_{n-1}$ are determined, (2.3) is a linear equation for $u_{n}$. The series (1.4) is constructed by solving this sequence of equations.

All of the $u_{n}$ 's are assumed to satisfy the boundary conditions

$$
u_{n}(0, t)=u_{n}(\pi, t)=0, \quad n \geq 1
$$

$u_{1}$ is assumed to satisfy the initial condition

$$
u_{1}(x, 0)=f(x)
$$

and the remaining $u_{n}$ 's are assumed to be zero initially:

$$
u_{n}(x, 0)=0, \quad n \geq 2 .
$$

The solution of (2.2), (2.4), and (2.5) is

$$
u_{1}(x, t)=\int_{0}^{\pi} f(\xi) G(x, \xi, t) d \xi
$$

where $G$ is Green's function given by

$$
G(x, \xi, t)=\frac{2}{\pi} \sum_{j=1}^{\infty} e^{-j^{2} t} \sin j \xi \sin j x .
$$


Once $u_{1}, u_{2}, \ldots, u_{n-1}$ are known, (2.3), (2.4), and (2.6) can be solved for $u_{n}$ to obtain

$$
u_{n}(x, t)=\int_{0}^{t} \int_{0}^{\pi} h_{n}(\xi, \tau) G(x, \xi, t-\tau) d \xi d \tau,
$$

for $n \geq 2$, where

$$
h_{n}(x, t)=\gamma \sum_{k=1}^{n-1} u_{n-k} u_{k}
$$

The formulation (2.8) of $G$ is useful for long-time estimates of the solution; for time-local estimates, we will use

$$
G(x, \xi, t)=\frac{1}{\sqrt{4 \pi t}} \sum_{n=-\infty}^{\infty}\left[\exp \left(-\frac{(x-\xi-2 \pi n)^{2}}{4 t}\right)-\exp \left(-\frac{(x+\xi-2 \pi n)^{2}}{4 t}\right)\right] .
$$

(See [3].)

\section{Convergence results}

Lemma 3.1. Let $\mu_{n}$ be a sequence of positive numbers defined by

$$
\mu_{n}=\kappa \sum_{k=1}^{n-1} \mu_{n-k} \mu_{k}
$$

where $\kappa>0$. Then

$$
\mu_{n} \leq\left(4 \kappa \mu_{1}\right)^{n}
$$

Proof. We repeat the argument given in [2] using the method of generating functions. Define

$$
\rho(z)=\kappa \sum_{n=1}^{\infty} \mu_{n} z^{n}
$$

from which follows

$$
\rho(z)^{2}=\kappa^{2} \sum_{n \geq 2} z^{n} \sum_{k=1}^{n-1} \mu_{n-k} \mu_{k}=\kappa \sum_{n \geq 2} z^{n} \mu_{n}=\rho(z)-\kappa \mu_{1} z .
$$

In other words, the generating function $\rho$ satisfies the quadratic

$$
\rho^{2}-\rho+\kappa \mu_{1} z=0,
$$

and so,

$$
\rho=\frac{1}{2} \pm \frac{1}{2} \sqrt{1-4 \kappa \mu_{1} z}
$$


The fact that $\mu_{n} \geq 0$ leads us to choose $\rho=1 / 2-(1 / 2) \sqrt{1-4 \kappa \mu_{1} z}$. Thus,

$$
\begin{aligned}
\rho^{(n)}(z) & =\left(-\frac{1}{2}\right)\left(\frac{1}{2}\right)\left(\frac{1}{2}-1\right) \cdots\left(\frac{1}{2}-n+1\right)\left(1-4 \kappa \mu_{1} z\right)^{(1 / 2-n)}\left(-4 \kappa \mu_{1}\right)^{n} \\
& =-\left(\frac{1}{2}\right)\left(-\frac{1}{2}\right)\left(\frac{1}{2}\right)\left(\frac{3}{2}\right)\left(\frac{5}{2}\right) \cdots\left(n-\frac{3}{2}\right)\left(1-4 \kappa \mu_{1} z\right)^{(1 / 2-n)}\left(4 \kappa \mu_{1}\right)^{n} \\
& =-\frac{1}{2}\left(1-4 \kappa \mu_{1} z\right)^{(1 / 2-n)}\left(4 \kappa \mu_{1}\right)^{n} \prod_{k=1}^{n}\left(k-\frac{3}{2}\right), \\
\mu_{n} & =\frac{\rho^{(n)}(0)}{n !}=-\frac{1}{2}\left(4 \kappa \mu_{1}\right)^{n} \prod_{k=1}^{n}\left(1-\frac{3}{2 k}\right)<\left(4 \kappa \mu_{1}\right)^{n} .
\end{aligned}
$$

In what follows, we adopt the following norm notation:

$$
\|\cdot\|_{\pi}=\sup _{0<x<\pi}|\cdot|, \quad\|\cdot\|_{\delta}=\sup _{\substack{0<x<\pi \\ 0<t<\delta}}|\cdot|, \quad\|\cdot\|=\sup _{\substack{0<x<\pi \\ t>0}}|\cdot| .
$$

Our first theorem concerns local convergence of (1.4). Specifically, we show that the time interval over which the series converges to a solution of (1.1), (1.2), and (1.3) is inversely related to the size of the initial data and the coefficient of the nonlinear term.

Theorem 3.2. With $u_{n}$ defined by (2.7) and (2.9), the series (1.4) converges uniformly on $[0, \pi] \times[0, \delta]$ if $\delta<1 /\left(4 \gamma\|f\|_{\pi}\right)$ and $f \in C([0, \pi])$.

Proof. It is not hard to show from (2.9) and (2.11) that $u_{n}$ can be expressed in the form

$$
u_{n}(x, t)=\int_{0}^{t} \int_{-\infty}^{\infty} \tilde{h}_{n}(\xi, \tau) H(x-\xi, t-\tau) d \xi d \tau
$$

where $\tilde{h}_{n}$ is the odd, $2 \pi$-periodic extension of $h_{n}$ and $H$ is the usual heat kernel,

$$
H(x, t)=\frac{\exp \left(-x^{2} / 4 t\right)}{\sqrt{4 \pi t}} .
$$

Then,

$$
\left\|u_{n}\right\|_{\delta} \leq\left\|h_{n}\right\|_{\delta} \int_{0}^{\delta} \int_{-\infty}^{\infty} H(x-\xi, \delta-\tau) d \xi d \tau=\delta\left\|h_{n}\right\|_{\delta}
$$

for $n \geq 2$. Combining this with (2.10), we have

$$
\left\|u_{n}\right\|_{\delta} \leq \gamma \delta\left\|\sum_{k=1}^{n-1} u_{n-k} u_{k}\right\|_{\delta} \leq \gamma \delta \sum_{k=1}^{n-1}\left\|u_{n-k}\right\|\left\|_{\delta}\right\| u_{k} \|_{\delta}
$$

for $n \geq 2$. By Lemma 3.1,

$$
\left\|u_{n}\right\|_{\delta} \leq\left(4 \gamma \delta\left\|u_{1}\right\|_{\delta}\right)^{n}
$$

From (2.7) and (2.11), we can argue that

$$
\left\|u_{1}\right\|_{\delta} \leq\|f\|_{\pi}
$$


which, with (3.13), implies that

$$
\left\|u_{n}\right\|_{\delta} \leq\left(4 \gamma \delta\|f\|_{\pi}\right)^{n}
$$

Thus (1.4) converges if $\delta<1 /\left(4 \gamma\|f\|_{\pi}\right)$.

Our next theorem gives conditions under which (1.4) converges globally.

Theorem 3.3. With $u_{n}$ defined by (2.7) and (2.9), the series (1.4) converges uniformly on $[0, \pi] \times[0, \infty)$ if $\|f\|_{\pi}<3 /\left(4 \gamma \pi^{2}\right)$.

Proof. Let

$$
K(s)=\sup _{0<x<\pi} \int_{0}^{\pi}|G(x, \xi, s)| d \xi
$$

Then by (2.9),

$$
\left\|u_{n}\right\| \leq\left\|h_{n}\right\| \int_{0}^{\infty} K(\tau) d \tau
$$

Notice from (2.8) that

$$
K(s) \leq \sup _{0<x<\pi} \frac{2}{\pi} \sum_{j=1}^{\infty} e^{-j^{2} s} \int_{0}^{\pi}|\sin j x \sin j \xi| d \xi \leq 2 \sum_{j=1}^{\infty} e^{-j^{2} s} .
$$

This means that

$$
\int_{0}^{\infty} K(s) d s \leq 2 \sum_{j=1}^{\infty} \frac{1}{j^{2}}=\frac{\pi^{2}}{3}
$$

Combining this with (3.17), we have

$$
\left\|u_{n}\right\| \leq \frac{1}{3} \pi^{2}\left\|h_{n}\right\| \leq \frac{1}{3} \gamma \pi^{2} \sum_{k=1}^{n-1}\left\|u_{n-k}\right\|\left\|u_{k}\right\| .
$$

Lemma 3.1 then implies that

$$
\left\|u_{n}\right\| \leq\left(\frac{4}{3} \gamma \pi^{2}\right)^{n}\left\|u_{1}\right\|^{n} \leq\left(\frac{4}{3} \gamma \pi^{2}\right)^{n}\|f\|_{\pi}^{n} .
$$

Thus (1.4) converges on $[0, \pi] \times[0, \infty)$ if $\|f\|_{\pi}<3 /\left(4 \gamma \pi^{2}\right)$.

\section{Neumann boundary conditions}

In this section, we replace (1.2) with Neumann boundary conditions, that is,

$$
\partial_{x} u(0, t)=\partial_{x} u(\pi, t)=0 .
$$


We now assume that the solution has the form

$$
u(x, t)=u_{0}(t)+\sum_{n=1}^{\infty} u_{n}(x, t)
$$

where $u_{0}$ is a purely time-dependent solution of (1.1), specifically,

$$
u_{0}(t)=\frac{a_{0}}{1-\gamma a_{0} t}
$$

where $a_{0}=(1 / \pi) \int_{0}^{\pi} f(x) d x$. (Notice that a global solution in time is not generally possible because of the blowup of $u_{0}$ in finite time.) Substituting (4.2) into (1.1) and using the fact that $u_{0}$ satisfies (1.1), we obtain

$$
\sum_{n=1}^{\infty} \partial_{t} u_{n}=\sum_{n=1}^{\infty} \partial_{x x} u_{n}+2 \gamma u_{0} \sum_{n=1}^{\infty} u_{n}+\gamma \sum_{n=2}^{\infty} \sum_{k=1}^{n-1} u_{n-k} u_{k}
$$

leading to the sequence of linear equations

$$
\begin{gathered}
\partial_{t} u_{1}=\partial_{x x} u_{1}+2 \gamma u_{0} u_{1}, \\
\partial_{t} u_{n}=\partial_{x x} u_{n}+2 \gamma u_{0} u_{n}+\gamma \sum_{k=1}^{n-1} u_{n-k} u_{k}, \quad n \geq 2 .
\end{gathered}
$$

We assume (2.6) as before, but now (2.5) is replaced by

$$
u_{1}(x, 0)=f(x)-u_{0}(0)=f(x)-a_{0}
$$

and (2.4) by

$$
\partial_{x} u_{n}(0, t)=\partial_{x} u_{n}(\pi, t)=0 .
$$

Expressed in terms of a Green's function, the solutions of (4.5), (4.8), (4.7), and (4.6), (4.8), (2.6) are

$$
\begin{gathered}
u_{1}(x, t)=\int_{0}^{\pi} \frac{\left(f(\xi)-a_{0}\right) G(x, \xi, t)}{\left(1-\gamma a_{0} t\right)^{2}} d \xi, \\
u_{n}(x, t)=\int_{0}^{t} \int_{0}^{\pi} h_{n}(\xi, \tau)\left(\frac{1-\gamma a_{0} \tau}{1-\gamma a_{0} t}\right)^{2} G(x, \xi, t-\tau) d \xi d \tau, \quad n \geq 2,
\end{gathered}
$$

where

$$
G(x, \xi, t)=\frac{1}{\sqrt{4 \pi t}} \sum_{n=-\infty}^{\infty}\left[\exp \left(-\frac{(x-\xi-2 \pi n)^{2}}{4 t}\right)+\exp \left(-\frac{(x+\xi-2 \pi n)^{2}}{4 t}\right)\right] .
$$

Theorem 4.1. With $u_{n}$ defined by (4.3), (4.9), and (4.10), the series (4.2) converges uniformly on $[0, \pi] \times[0, \delta]$ if $\left\|f-a_{0}\right\|_{\pi}<\left(1-\gamma a_{0} \delta\right)^{4} /(4 \gamma \delta)$ and $f \in C([0, \pi])$. 
Proof. Equations (4.10) and (4.11) can be used to express $u_{n}$ in the form

$$
u_{n}(x, t)=\int_{0}^{t} \int_{-\infty}^{\infty} \tilde{h}_{n}(\xi, \tau)\left(\frac{1-\gamma a_{0} \tau}{1-\gamma a_{0} t}\right)^{2} H(x-\xi, t-\tau) d \xi d \tau,
$$

where $\tilde{h}_{n}$ is the even, $2 \pi$-periodic extension of $h_{n}$ and $H$ is defined by (3.10). Then,

$$
\begin{aligned}
\left\|u_{n}\right\|_{\delta} & \leq\left\|h_{n}\right\|_{\delta} \int_{0}^{\delta}\left(\frac{1-\gamma a_{0} \tau}{1-\gamma a_{0} \delta}\right)^{2} \int_{-\infty}^{\infty} H(x-\xi, \delta-\tau) d \xi d \tau \\
& =\left\|h_{n}\right\|_{\delta} \int_{0}^{\delta}\left(\frac{1-\gamma a_{0} \tau}{1-\gamma a_{0} \delta}\right)^{2} d \tau \leq \frac{\delta\left\|h_{n}\right\|_{\delta}}{\left(1-\gamma a_{0} \delta\right)^{2}}
\end{aligned}
$$

for $n \geq 2$. Combining this with (2.10), we have

$$
\left\|u_{n}\right\|_{\delta} \leq \frac{\gamma \delta}{\left(1-\gamma a_{0} \delta\right)^{2}}\left\|\sum_{k=1}^{n-1} u_{n-k} u_{k}\right\|_{\delta} \leq \frac{\gamma \delta}{\left(1-\gamma a_{0} \delta\right)^{2}} \sum_{k=1}^{n-1}\left\|u_{n-k}\right\|\left\|_{\delta}\right\| u_{k} \|_{\delta}
$$

for $n \geq 2$. By Lemma 3.1,

$$
\left\|u_{n}\right\|_{\delta} \leq\left(\frac{4 \gamma \delta\left\|u_{1}\right\|_{\delta}}{\left(1-\gamma a_{0} \delta\right)^{2}}\right)^{n}
$$

From (4.9) and (4.11), we can argue that

$$
\left\|u_{1}\right\|_{\delta} \leq \frac{\left\|f-a_{0}\right\|_{\pi}}{\left(1-\gamma a_{0} \delta\right)^{2}}
$$

which, with (4.15), implies that

$$
\left\|u_{n}\right\|_{\delta} \leq\left(\frac{4 \gamma \delta\left\|f-a_{0}\right\|_{\pi}}{\left(1-\gamma a_{0} \delta\right)^{4}}\right)^{n}
$$

Thus (1.4) converges if

$$
\left\|f-a_{0}\right\|_{\pi}<\frac{\left(1-\gamma a_{0} \delta\right)^{4}}{4 \gamma \delta}
$$

\section{References}

[1] G. Adomian, Solving Frontier Problems of Physics: The Decomposition Method, Fundamental Theories of Physics, vol. 60, Kluwer Academic Publishers, Dordrecht, 1994.

[2] A. Boumenir and M. Gordon, The rate of convergence for the decomposition method, Numer. Funct. Anal. Optim. 25 (2004), no. 1-2, 15-25.

[3] R. Haberman, Applied Partial Differential Equations, 4th ed., Prentice-Hall, New Jersey, 2004.

Michael S. Gordon: Department of Mathematics, College of Arts and Sciences, University of West Georgia, Carrollton, GA 30118, USA

E-mail address: sgordon@westga.edu 


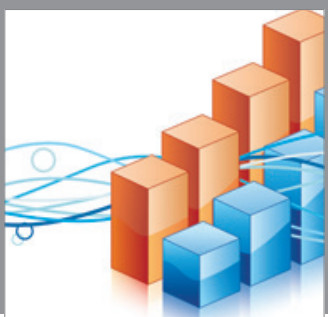

Advances in

Operations Research

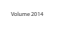

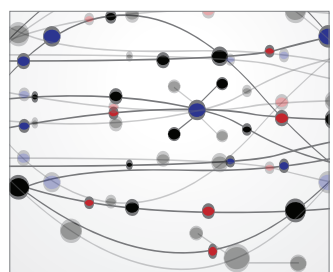

\section{The Scientific} World Journal
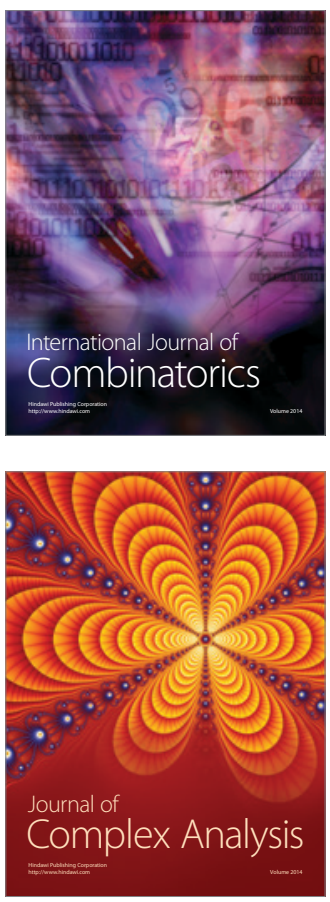

International Journal of

Mathematics and

Mathematical

Sciences
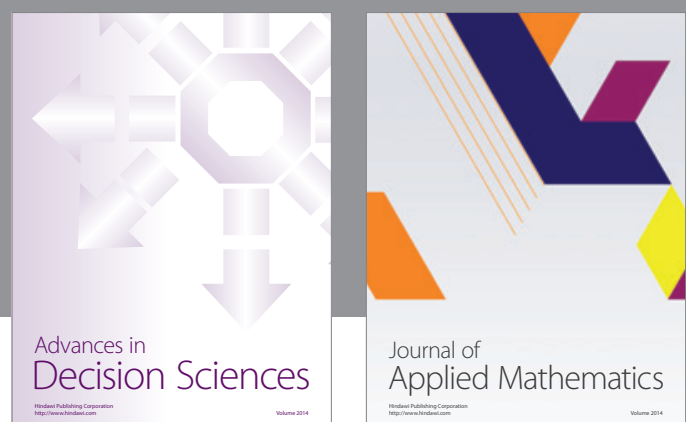

Journal of

Applied Mathematics
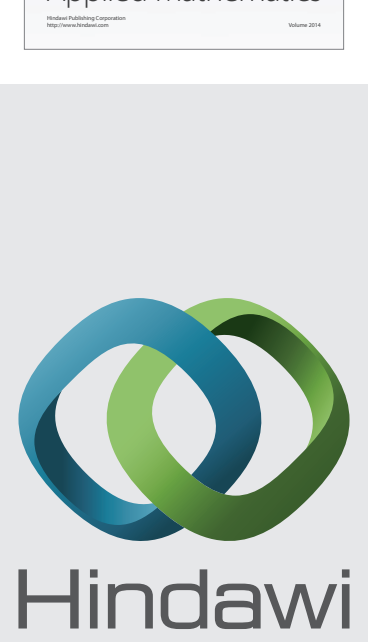

Submit your manuscripts at http://www.hindawi.com
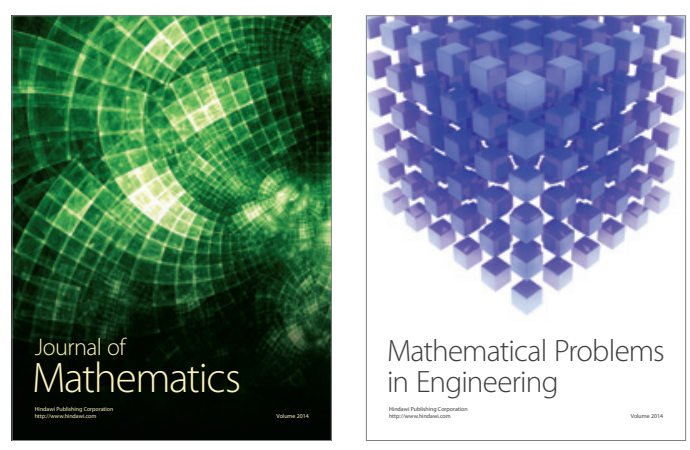

Mathematical Problems in Engineering
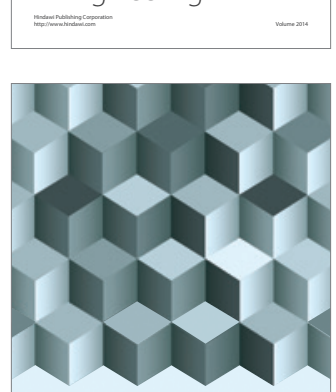

Journal of

Function Spaces
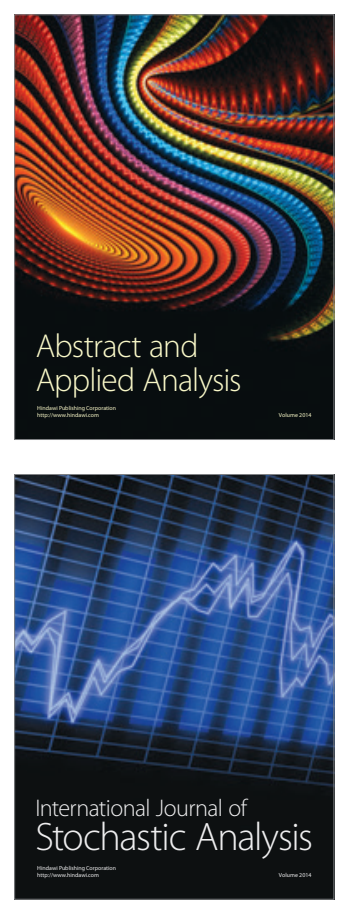

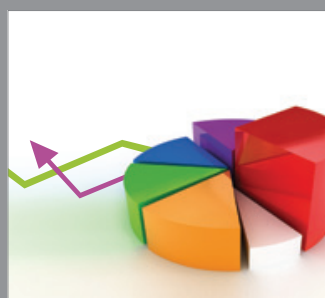

ournal of

Probability and Statistics

Promensencen
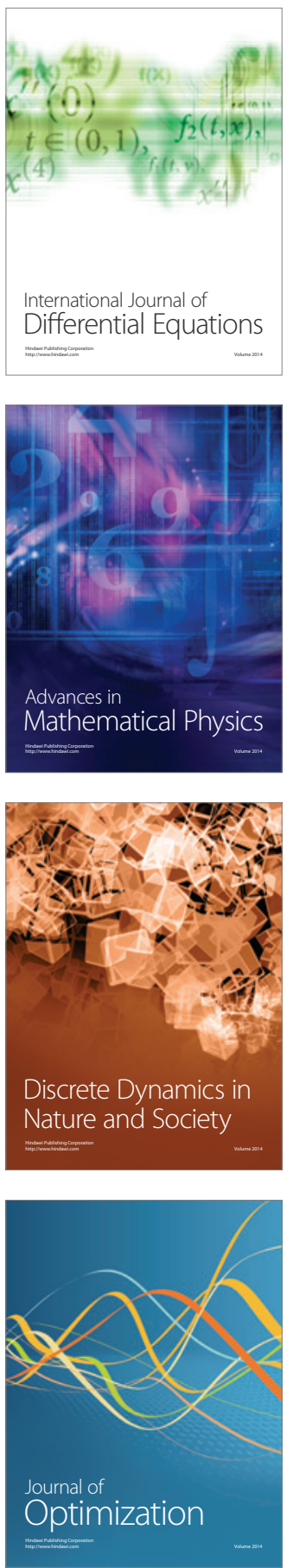